\title{
Diverse roles of reduced learning ability of players in evolution of cooperation
}

\author{
Zhi-Xi Wu ${ }^{1,2}$, Zhihai Rong ${ }^{3,4}$ and Michael Z. Q. Chen ${ }^{2}$ (a) \\ 1 Institute of Computational Physics and Complex Systems, Lanzhou University, Lanzhou Gansu 730000, People's \\ Republic of China \\ 2 Department of Mechanical Engineering, The University of Hong Kong, Pokfulam, Hong Kong \\ 3 CompleX Lab, Web Sciences Center, University of Electronic Science and Technology of China, Chengdu 610054, \\ People's Republic of China \\ 4 Department of Electronic and Information Engineering, The Hong Kong Polytechnic University, Hung Hom, \\ Kowloon, Hong Kong
}

\begin{abstract}
PACS 02.50.Le - Decision theory and game theory
PACS 07.05. Tp - Computer modeling and simulation

PACS 87.23.-n-Ecology and evolution
\end{abstract}

\begin{abstract}
Individual heterogeneity in reproductive rate is found to play an important role in the emergence and persistence of cooperation. Most existing literature focused mainly on the enhancement of cooperation by the introduction of inhomogeneous teaching capability of the individuals. It is far from clear how heterogeneous learning ability of the individuals affects the evolution of cooperation. To fill this research gap, we make comparative studies of the evolutionary spatial prisoner's dilemma game with reduced learning or teaching ability of the players, under both synchronous and asynchronous strategy updating schemes. By carrying out extensive computer simulations, we show that cooperation can always be facilitated if inhomogeneous teaching ability of the players is considered, irrespective of the strategy updating manner. By contrast, cooperation is promoted (inhibited) in the case of synchronous (asynchronous) strategy updating, if heterogeneous learning ability is considered, which is attributed to the reduced ability of cooperators to expand their domains.
\end{abstract}

Introduction. - Cooperation is ubiquitous in nature, ranging from biological, social, physical, to economical systems [1]. Understanding and searching for generic mechanisms that can foster and sustain cooperation among selfish, unrelated individuals still remains an interesting and challenging problem up to date [2-5]. Evolutionary game theory offers a powerful mathematical framework to address the conundrum of evolution of cooperation [2]. Particularly, the prisoner's dilemma (PD) has long been considered the paradigm for studying the emergence of cooperation among selfish individuals. In a typical scenario of the PD game, two interacting individuals have to choose simultaneously whether they want to cooperate or defect. Being a cooperator will pay the cost $c$ in order to provide a greater benefit $b$ to the partner. Being a defector does not distribute any benefits and incur no cost. Under such circumstances, mutual cooperation will

(a) corresponding author: mzqchen@hku.hk yield the highest collective benefit shared equally between the players, while a defector can have a higher individual payoff if the opponent decides to cooperate. Therefore, mutual cooperation can easily be crushed by any unilateral defection, which in turn drives both players to choosing defection, whereby they will end up with a lower payoff than if both would cooperate, leading to the deadlock and dilemma.

In the past decades, many mechanisms supporting cooperation have been proposed to resolve the dilemma, such as kin selection [6], direct and indirect reciprocity $[7,8]$, spatial (or network) reciprocity [9, 10], multi-level (or group) selection [11], voluntary participation [12], nonlinear attractive effect [13], dynamical linking [14], migration [15], credit records [16], co-evolving networks [17], diversity of time scale $[18,19]$, heterogeneous interaction degree [20], interdependent interaction network [21], punishment [22, 23], wisdom of group [24], to name a few. 
For a comprehensive review on this field, we refer to Refs. [25, 26], and for some recent brief reviews, we refer to Refs. [27, 28].

One of the most recent contributions to the subject is concerned with the assignment of heterogeneous imitation capability to players such that different individuals may respond differently to the same payoff information, representing the diversity of human behavior (or social status) in reality [29]. It is noticeable that when the inhomogeneous imitation activity is introduced, either by artificially adding unidirectional connections [30] or through the heterogenous interaction degree [31], to characterize the asymmetric and different influence of players to each other, remarkable increase of cooperation can be observed. $\mathrm{Wu}$ et al. introduced dynamic impact weight among the interacting individuals, guiding the preferential selection of a neighbor whose strategy would be imitated [32]. The time evolution of impact weight is according to the rule of "win-strengthen, lose-weaken". The authors found that cooperative behavior can be remarkably promoted, owing to the formed heterogeneous impact weight distribution in the steady state (through which compact clusters of cooperators can be sustained stably). In Ref. [33], Szolnoki and Szabó studied a simplified version of the model introduced in [32], and considered just two types of players, say $A$ and $B$, with one type having reduced teaching activity in exerting strategies on others. By studying the concentration of cooperators as a function of the fraction of individuals with reduced teaching activity, the authors found that the diversity in the teaching capability can enforce the emergence of cooperative behavior among selfish competitors. In their further study [34], it was revealed that the mechanism for the promotion of cooperation is conceptually similar to the one reported previously for scale-free networks by Santos and Pacheco [31].

Though the role of asymmetric teaching activity in sustaining cooperation has already been investigated exhaustively in the literature [33,34], how asymmetric learning activity of the individuals affects the evolution of cooperation remains largely unexplored. Moreover, we notice that in the previous work by Szolnoki et al. [33,34], the strategy updating process was usually implemented asynchronously. It has been pointed out that whether strategy updating is implemented synchronously or asynchronously may play a considerable role in determining the quantitative, or even qualitative, properties of the final evolutionary outcomes. In Ref. [35], Huberman and Glance discovered for the first time that large disparities may occur by using the two different strategy updating scenarios in cellular automata-type simulations for the study of cooperation. When a stochastic imitation process characterized by the Fermi function is adopted for the update rule [36-38], the synchronous strategy updating manner usually leads to a lower critical value of the temptation to defect, beyond which cooperators are wiped out in the population $[32,39]$. More recently, we have shown that when the game evolving dynamics is governed by an aspiration-based response rule [40], intermediate aspirations will give rise to the lowest cooperation level [41], in contrast to the highest cooperation level obtained when an asynchronous strategy updating scheme is employed instead [40].

In this paper, we wish to extend the scope of asymmetric effects on the evolution of cooperation in the spatial PD game by introducing inhomogeneous imitation activities of players as their intrinsically determined property. For this purpose, we will adopt the approach of Refs. [33,34] to exploring the consequence of varying learning and teaching activity in the strategy imitation process, through carrying out both synchronous and asynchronous strategy updating of the game dynamics. As we will show below that, different from the claim in [33], inhomogeneous learning activity does have relevant and significant impact on the evolution of cooperation in spatial PD game.

Model. - We consider an evolutionary two-strategy PD game with players located on vertices of either a square lattice with periodic boundary conditions or random regular graphs (each vertex has a degree of 4). Without loss of generality, we focus on the donation game, which is a true PD game, according to which the elements of the payoff matrix are: reward for mutual cooperation 1.0, the temptation to defect $b=1+c$ (where $0<c<1$ ), punishment for mutual defection 0 , and the sucker's payoff $-c$. Note that the weak PD version was considered in [33,34], where the above sucker's payoff is set to zero.

In the initial stage, each individual can either choose to cooperate or to defect with equal probability. Following $[33,34]$, amongst all players, a fraction $\nu$ of players are chosen randomly and designated as having a restricted ability to transfer their strategy or learn from others, irrespective of their initial strategies. After that, we simulate the evolutionary process in accordance with the standard Monte Carlo (MC) simulation procedure comprising the following elementary steps. In the asynchronous strategy updating scheme, we randomly select a player $x$, among the population, to collect its payoff $P_{x}$ by playing the game with all its neighbors. Next, one neighbor $y$ of the player $x$ is also randomly chosen, and acquires its payoff $P_{y}$ in the same way as previously the player $x$. Finally, the player $x$ adopts the strategy from the player $y$ with a probability $q$ in dependence of their payoff difference, which is determined by the Fermi function

$$
q=w_{x} \frac{1}{1+\exp \left[\left(P_{x}-P_{y}\right) / \kappa\right]},
$$

in the case of learning-activity-driven strategy updating, or by

$$
q=w_{y} \frac{1}{1+\exp \left[\left(P_{x}-P_{y}\right) / \kappa\right]},
$$

in the case of teaching-activity-driven strategy updating. The values of pre-factors $w_{x}, w_{y}$ can be either 1 or $w$, whose value is restricted in the region $(0,1)$, characterizing the reduced learning or teaching activity of the players. The parameter $\kappa$ denotes the amplitude of noise [36-38], 
or selection intensity [42], related to the strategy adoption process. Positive values of $\kappa$ imply that better performing players are readily imitated, but it is not impossible to adopt the strategy of a player who is performing worse. Such errors in judgment can be attributed to mistakes and external influences that affect the evaluation of the opponent. In the present study, $\kappa$ is set to be 0.4 , a value that was advantageous for cooperation in the scenario with $w_{x}=w_{y}=1$ [43]. During a full MC step, all players will have a chance to pass their strategy once on average. In the synchronous strategy updating scheme, rather than sequentially selecting individuals to revise strategies, all the players acquire payoffs by playing with their neighbors and update their strategies simultaneously according to Eq. (1) or Eq. (2), depending on whether learning or teaching activity driven strategy updating is under consideration.

Unless otherwise specified, all our simulations are performed in the population of size $N=250 \times 250$. In each independent run, the key quantity, i.e., the average fraction of cooperators $f_{C}$, is obtained by averaging over $10^{4}$ $\mathrm{MC}$ steps after a long relaxation of $3 \times 10^{4}$ steps to ensure the dynamical equilibrium has been convincingly achieved. The data presented below are averaged over up to 30 independent runs for each set of parameter values in order to assure suitable accuracy.

It is worth pointing out that the extended mean-field method exemplified in [25], i.e., pair approximation, based on the assumption of continuous time to address the asynchronous strategy updating dynamics, cannot distinguish the effect of reduced learning and teaching activities, since the pre-factors $w_{x}$ and $w_{y}$ can be absorbed into the time factor, and hence the numerical solutions of Eqs. (1) and (2) remain unchanged for any positive $w$. For this reason, we mainly resort to MC simulations and qualitative analysis in our current studies.

Results and analysis. - Let us first discuss the computer simulation results of our model defined on a square lattice. In Fig. 1, we have plotted the average fraction of cooperators $f_{C}$ in the steady state as a function of $\nu$ for $b=1.02$ and different values of $w=1.0,0.3,0.1$ and 0.05 . In particular, Figs. 1(a) and 1(b) [ 1(c) and 1(d)] correspond to the case of reduced learning (teaching) activity of the players, while the left column[Figs. 1(a) and 1(c)] and right column [Figs. 1(b) and 1(d)] of Fig. 1 are for the synchronous and asynchronous strategy updating schemes, respectively.

Note that for $w=1.0$, the model is equivalent to a homogeneous system, where $f_{C}$ will not change with $\nu$. In addition, for $\nu=0$ and $\nu=1$, no matter what the value of $w$ is, we actually consider systems with the same evolutionary dynamics (albeit with different evolving time scales). Hence we will pay more attention to the cases of $w<1.0$. Note that $w=0$ will not be considered, since the system does not evolve at all.

Reduced teaching activity with asynchronous strategy
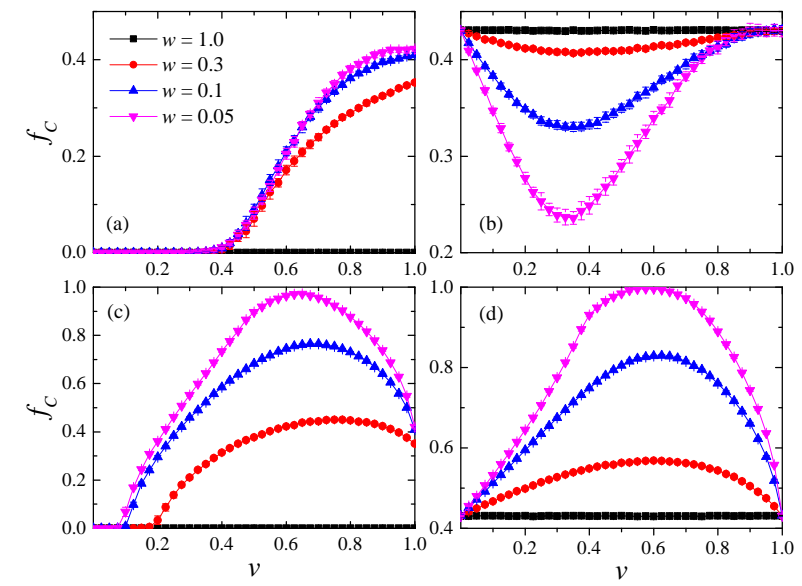

Fig. 1: Average fraction of cooperators $f_{C}$ in the stationary state in dependence of $\nu$ for different $w$, obtained for $b=1.02$ and $\kappa=0.4$. The top (bottom) two panels correspond, respectively, to the cases of reduced learning (teaching) activity, and the left (right) columns correspond to synchronous (asynchronous) strategy updating, respectively. Each data point is averaged over 30 independent runs, and the error bars are smaller or comparable to the symbol size. Note the different scales of the axis.

updating scheme. Basically, the properties of our obtained results [Fig. 1(d)] in such a situation are similar to those found by Szolnoki et al. for the weak PD game [33,34]: For all $w<1, f_{C}$ increases monotonically until reaching the maximum value at some moderate value of $\nu$, and then decreases to the levels at $\nu=0$. And for a sufficiently small $w=0.05, f_{C}$ can even reach unity, that is, cooperators can completely dominate the population. It was revealed that when the teaching activity is reduced from a moderate fraction of the players (distributed randomly among the population), some influential cooperators (with $w_{x}=1.0$ ) may grow as local "leaders" to attract others to imitate them, which will stabilize the compact clusters composed of cooperators, and further facilitate the development of a high level of cooperation.

Reduced teaching activity with synchronous strategy updating scheme. In such a case, the properties of the results [Fig. 1(c)] are generally similar to the case of asynchronous strategy updating: The presence of moderate fraction of players with reduced teaching activity benefits the emergence of cooperation the most, and for each $\nu$, smaller $w$ usually results in higher $f_{C}$. The main difference comes from the extreme case $\nu=1.0$, where $f_{C}$ is now greater than that for $\nu=0.0$, in stark contrast to asynchronous case Fig. $1(\mathrm{~d})$. We notice that for $\nu=1.0$, as $w$ is decreasing, the effective number of players who succeed in strategy transformation is also becoming less so. As a result, for a very small $w$, the relaxation becomes sufficiently slow such that the system with synchronous strategy updating can be regarded as being equivalent to the one with asynchronous updating. This point can be verified by the fact that $f_{C} \simeq 0.42$ for $\nu=1.0$ and $w=0.05$ in Fig. 1(c), 

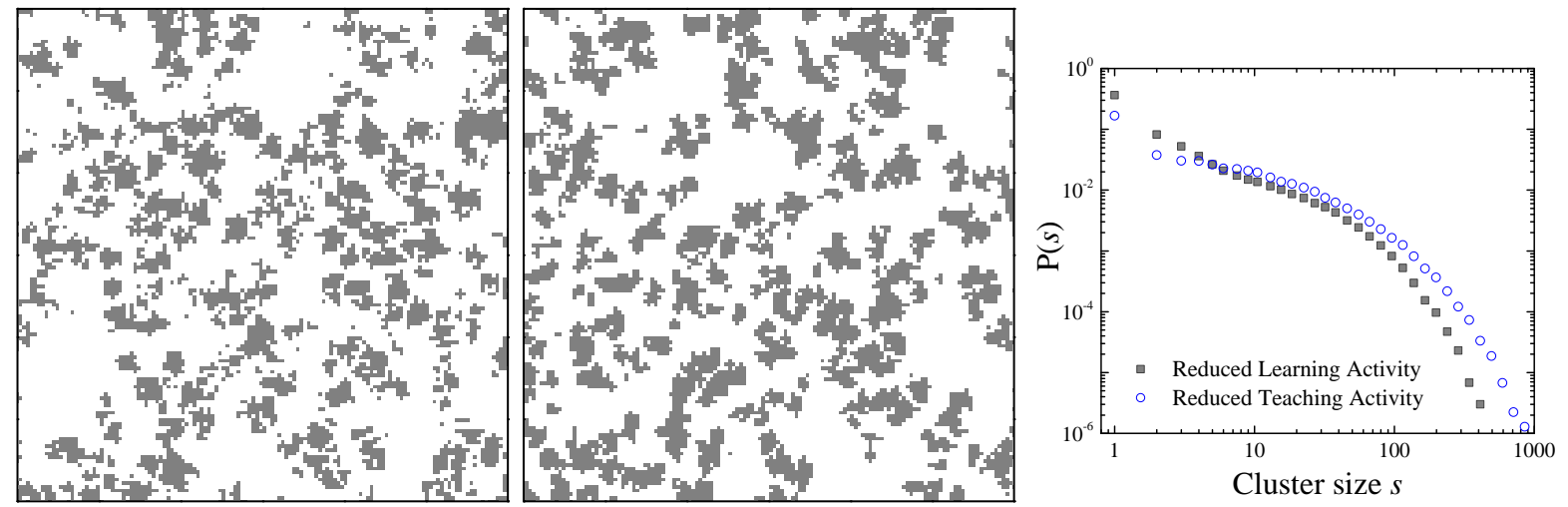

Fig. 2: Typical snapshots of the strategy configuration of the individuals in the stationary state by employing asynchronous upadting, and cooperators and defectors are colored by dark gray and white, respectively. Left panel (middle panel) is for the case of reduced learning (teaching) activity with $b=1.02(b=1.075), \nu=0.5$, and $w=0.05$. The average $f_{C}$ in the steady state are around $0.287(5)$, and the average cluster size of cooperators are about 18 and 39 , for the two cases. Note that only a fraction of the total $250 \times 250$ lattice is displayed. Right panel illustrates the corresponding cluster size distribution of cooperators in the equilibrium for the two considered cases.
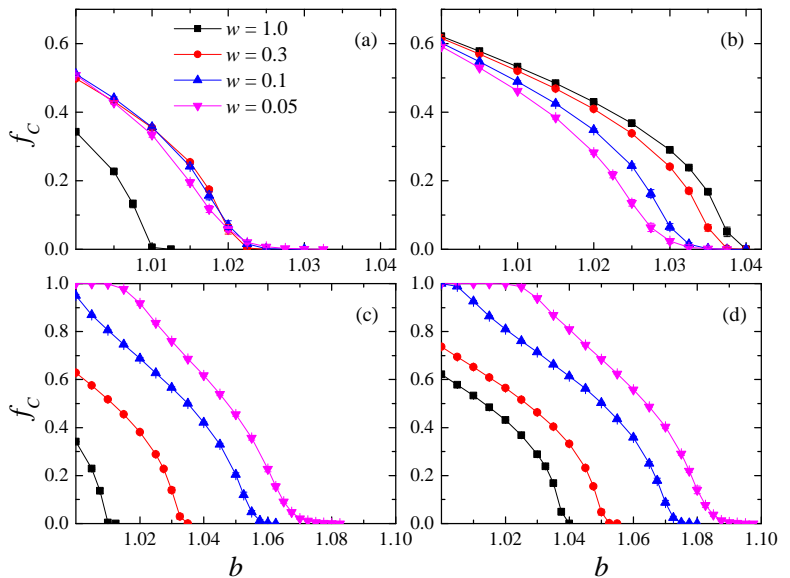

Fig. 3: Average fraction of cooperators $f_{C}$ in the stationary state as a function of the temptation $b$ for different $w$, obtained for $\nu=0.5$ and $\kappa=0.4$. The top (bottom) two panels correspond, respectively, to the cases of reduced learning (teaching) activity, and the left (right) columns correspond to synchronous (asynchronous) strategy updating, respectively. Each data point is averaged over from 30 independent runs, and the error bars are smaller or comparable to the symbol size. Note the different scales of the axis.

which is in consistent with $f_{C} \simeq 0.43$ for $\nu=0$ or 1.0 in Fig. 1(d).

Reduced learning activity with asynchronous strategy updating scheme. In this situation, we observe in Fig. 1(b) that $f_{C}$ varies with $\nu$ in a totally different way from that in the case of reduced teaching activity [Fig. 1(d)]. In particular, for different $w$, as $\nu$ increases from zero to unity, $f_{C}$ decreases monotoniclly until reaching the minimum value at some moderate value of $\nu$ and then goes up to the levels at $\nu=0$. Rather than promoting the evolution of cooperation in the case of reduced teaching activity, the
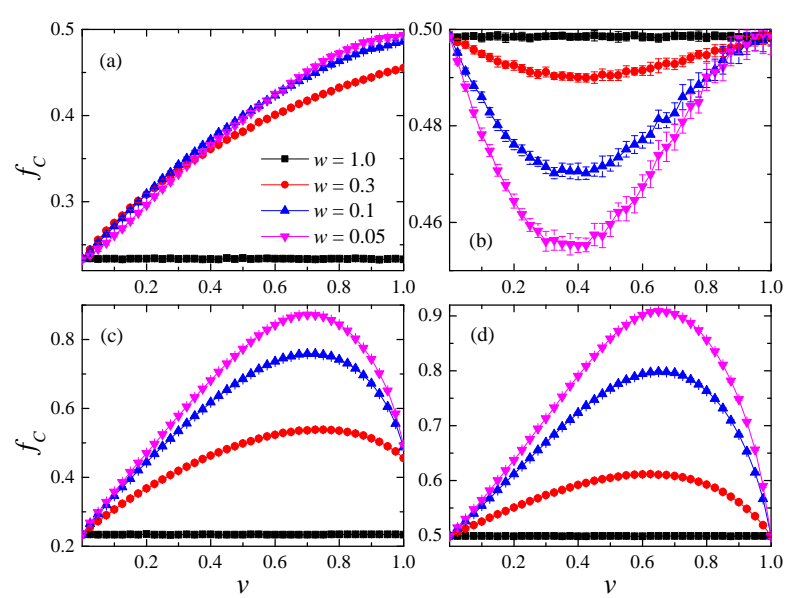

Fig. 4: Same as in Fig. 1, but for the PD game taking place on random regular graphs.

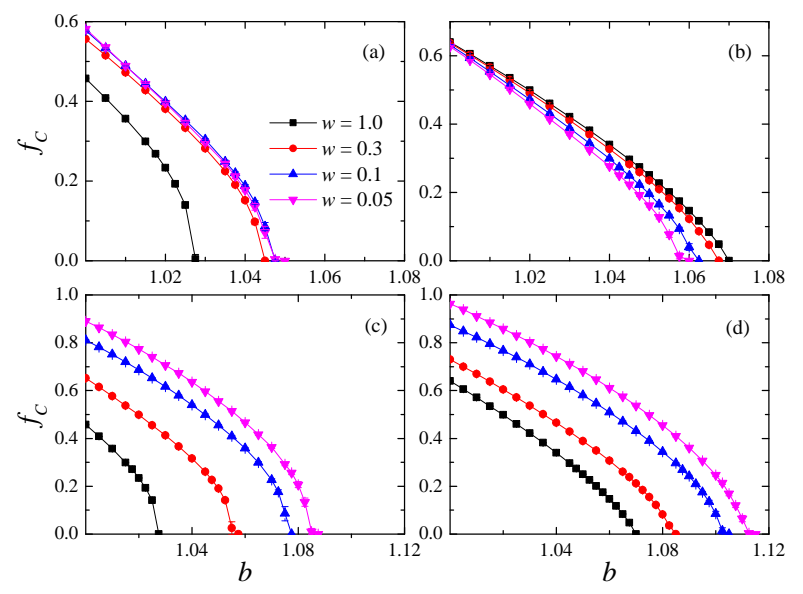

Fig. 5: Same as in Fig. 3, but for the PD game taking place on random regular graphs. 
introduction of the inhomogeneous learning activity of the players inhibits the formation and boom of cooperation. In Ref. [33], the authors mentioned that cooperation is not modified directly by the application of inhomogeneous learning activities for $w=0.1$ and $\nu=0.5$. Here, different from their claims, we do find that inhomogeneous learning activities of the players play a significant role in determining the final cooperation level. And, as shown in Fig. 1(b), it is actually detrimental to cooperation under asynchronous strategy updating.

How do inhomogeneous learning and teaching abilities give rise to totally contradicting outcomes? Let us first figure out how these mechanisms work at the microscopic level of the players' strategy transformation. When the game dynamics is driven by Eq. (2), a fraction $\nu$ of the players are assigned with reduced teaching activity, which actually means that the remaining fraction $1-\nu$ are specified with enhanced teaching capability. Whenever the players revise their strategies, those "influential" players with enhanced teaching ability have a greater impact on their neighbors so that their strategies are more likely to be imitated, in comparison with the players with reduced teaching activity. Consequently, if at the initial stage, some influential cooperators happened to have enough cooperative neighbors, they would attract their "weak" defective neighbors to follow them to form stable clusters of cooperators. From Fig. 1(d), we note that the best condition for cooperation arises at $\nu \simeq 0.7$.

However, when the game dynamics is guided by Eq. (1), being "influential" or "weak" players becomes less important, since the pre-factor $w_{x}$ in Eq. (1) just decreases the possibility of strategy transformation of one's own, and exerts no impact on immediate neighbors. In fact, under such circumstances, it is more difficult for cooperators to grow into large-size clusters in comparison with the case of reduced teaching activity, since those surrounding "weak" defectors of a cluster (composed of cooperators) have a negligible ability (or willingness) to change to cooperation provided $w$ is very small. This argument is validated by the results in Fig. 2, where the typical strategy configurations of the players in both cases of reduced learning and teaching activities are shown for comparison. We have calibrated parameters such that the stationary $f_{C}$ for the two cases are nearly the same. It is shown that cooperators can form more compact clusters when reduced teaching activity is considered, which is further substantiated by analyzing the cluster size distribution of cooperators in the equilibrium. As demonstrated by the curves in the right panel of Fig. 2, cooperators are usually able to form clusters with larger sizes in the case of reduced teaching activity. On the other hand, the presence of "slow-witted" defectors (e.g. with $w_{x}=0.05$ ) may easily induce their "quick-witted" cooperative neighbors (with $w_{x}=1.0$ ) to mimic them. Combining these two factors, it is foreseeable that cooperation might be considerably inhibited for moderate values of $\nu$ with small $w$ when reduced learning activity and asynchronous strategy updating is adopted.
Reduced learning activity with synchronous strategy updating scheme. From Fig. 1(b), we recognize that the combination of reduced learning activity with asynchronous strategy updating suppresses the development of cooperation. However, when the strategy updating is performed synchronously instead, cooperators regain advantage in expanding their territory in the presence of reduced learning capability. The results in Fig. 1(a) display clearly that as $\nu$ increases to unity, the concentration of cooperators also increases for all $w<1.0$. We note that the cooperation level saturates if the parameter $w$ is sufficiently small, e.g., $w<0.1$. Specifically, for $\nu=1.0$ (i.e., all the players are designated as having a restricted ability to learn from others), we once again observe that for small $w$, $f_{C} \simeq 0.42$, whose value is consistent with that in the case of asynchronous strategy updating with $\nu=1.0$, where $f_{C} \simeq 0.43$ in Fig. 1(b). Note the different scales of the $\mathrm{y}$-axis of the sub-figures.

The above phenomenon can be understood as follows. For $\nu=0$, all the players can respond relatively quickly to positive payoff differences. Since cooperators and defectors are distributed randomly in the initial state, defectors can earn higher payoffs than cooperators. As a result, the cooperation level will decrease monotonically as time evolves. If the temptation to defect $b$ is sufficiently small, the survived cooperators have a chance to strike back by forming compact clusters [9]. But if $b$ is large (e.g., $b=1.02$ in this case), defectors are able to invade the clusters of cooperators definitely and cooperators are doomed to extinction. With the introduction of players with reduced learning activities, the velocity of invasion of defectors on cooperators is considerably slowed down. When all the players try to update their strategies simultaneously, those already clustered cooperators may obtain a fair opportunity to enforce the neighboring "weak" defectors to follow them. In this way, cooperators may form stable clusters to support each other. Note that clustered defectors have much less likelihood to drive neighboring cooperators to follow them, since mutual defection results in just zero payoffs for the involved players.

Up to now, we have figured out how the combinations of strategy updating manners and reduced learning or teaching activities affect the stationary concentration of cooperators for a special value of the temptation to defect $b=1.02$. In order to obtain a thorough view on their combinative effects, we have in Fig. 3 featured the dependence of $f_{C}$ on $b$ for several $w$ values, with the parameter $\nu$ being fixed as 0.5 . The presented results show explicitly that when inhomogeneous teaching strategy is considered, cooperation can always be enhanced to some extent irrespective of the strategy updating manners [Figs. 3(c) and $3(\mathrm{~d})$ ]. However, when inhomogeneous learning activity is taken into account instead, how the strategy updating is executed has an essential impact on the evolution of cooperation. In particular, asynchronous strategy updating will deteriorate the flourish of cooperation, in stark contrast to the synchronous updating case, as reflected 
in Fig. 3(b), where with the decrease of $w$ the stationary concentration of cooperation $f_{C}$ is also decreased correspondingly for fixed $b$ values.

Conclusion and discussion. - To summarize, we have implemented extensive computer simulations to systematically investigate the role of reduced learning or teaching ability of the players in the evolution of cooperation in evolutionary spatial prisoner's dilemma games. Our main findings are: I) Reduced teaching ability of the players is beneficial for the promotion of cooperation irrespective of whether the strategy updating is implemented in a synchronous or asynchronous manner; II) Reduced learning ability of the players can foster the development of cooperation only in the case of synchronous strategy updating, and inhibit the evolution of cooperation provided the strategy updating is executed in the asynchronous manner.

To check whether the above findings are also valid for other complex underlying interaction structure, we have studied our model on random regular graphs [44], where the connections among the players are totally random, except that each player has exactly 4 neighbors. The results are summarized in Figs. 4 and 5. We see that, despite of some quantitative differences, all the qualitative properties of the results on square lattice are preserved. Thus, our findings are robust to the underlying interaction structure of the individuals. We believe that our current work has provided a thorough overview on how the asymmetry of learning and teaching activities affect the evolution of cooperation in spatially structured populations.

\section{$* * *$}

This work was partly supported by the National Natural Science Foundation of China (Nos. 11135001, 11475074,61374053 and 61473060), the Research Foundation of UESTC and Hong Kong Scholars Program (No. XJ2013019 and G-YZ4D).

\section{REFERENCES}

[1] R. Axelrod, The Evolution of Cooperation (Basic Books, New York) 1984

[2] J. W. Weibull, Evolutionary Game Theory (MIT Press, Cambridge, MA) 1995.

[3] R. Cressman, Evolutionary Dynamics and Extensive Form Games (MIT Press, Cambridge, MA) 2003.

[4] K. Sigmund, The Calculus of Selfishness (Princeton University Press, Princeton, MA) 2010.

[5] M. A. NowaK, Science, 314 (2006) 1560.

[6] W. D. Hamilton, J. Theor. Biol., 7 (1964) 1.

[7] R. L. Trivers, Q. Rev. Biol., 46 (1971) 35.

[8] M. A. Nowak ANd K. Sigmund, Nature, 437 (2005) 1291.

[9] M. A. NowaK AND R. M. May, Nature, 359 (1992) 826.

[10] H. Ohtsuki, C. Hauert, E. Lieberman, and M. A. NowAK, Nature, 441 (2006) 502.

[11] A. Traulsen and M. A. Nowak, Proc. Natl. Acad. Sci. USA, 103 (2006) 10952.
[12] G. Szabó and J. Vukov, Phys. Rev. E, 69 (2004) 036107.

[13] J.-Y. Guan, Z.-X. Wu, Z.-G. Huang, X.-J. Xu, Y.-H. WANG, EPL, 76 (2006) 1214.

[14] J. M. Pacheco, A. Traulsen, and M. A. Nowak, Phys. Rev. Lett., 97 (2006) 258103.

[15] H.-X. Yang, Z.-X. Wu, and B.-H. Wang, Phys. Rev. E, 81 (2010) 065101(R).

[16] C. Zhai, H.-T. Zhang, Y. Zhao, Michael Z. Q. Chen, Z.-H. Rong, And B.-H. Wang, Phys. Rev. E, 81 (2010) 036112

[17] A. Szolnoki And M. Perc, EPL, 86 (2009) 30007.

[18] Z. Rong, Z.-X. Wu, and G. Chen, EPL, 102 (2013) 68005.

[19] Z. Rong, Z.-X. Wu, D. HaO, Michael Z. Q. Chen, And T. Zhou, New J. Phys., 17 (2015) 033032.

[20] H.-X. YAnG, Z.-X. Wu, AND W.-B. Du, EPL, 99 (2012) 10006.

[21] Z. Wang, A. Szolnoki, and M. Perc, Sci. Rep., 3 (2013) 2470.

[22] X. Chen, A. Szolnoki, and M. Perc, New J. Phys., 16 (2014) 083016.

[23] H.-X. Yang, Z.-X. Wu, Z. Rong, and Y.-C. Lai, Phys. Rev. E, 91 (2015) 022121.

[24] A. Szolnoki, Z. Wang And M. Perc, Sci. Rep., 2 (2012) 576.

[25] G. Szabó and G. FÁth, Phys. Rep., 446 (2007) 97.

[26] M. A. NowAK, Evolutionary Dynamics (Harvard University, Cambridge, MA) 2006.

[27] M. Perc And A. Szolnoki, Biosystems, 99 (2010) 109.

[28] M. Perc, J. Gómez-Gardeñes, A. Szolnoki, L. M. Floría, and Y. Moreno, J. R. Soc. Interface, 10 (2013) 20120997.

[29] M. Perc And A. Szolnoki, Phys. Rev. E, 77 (2008) 011904.

[30] B. J. Kim, A. Trusina, P. Holme, P. Minnhagen, J. S. Chung, And M. Y. Choi, Phys. Rev. E, 66 (2002) 021907.

[31] F. C. Santos and J. M. Pacheco, Phys. Rev. Lett., 95 (2005) 098104.

[32] Z.-X. Wu, X.-J. Xu, Z.-G. Huang, S.-J. Wang, and Y.-H. WANG, Phys. Rev. E, 74 (2006) 021107.

[33] A. Szolnoki and G. Szabô, EPL, 77 (2007) 30004.

[34] A. Szolnoki, M. Perc, and G. Szabó, Eur. Phys. J. B, 61 (2008) 505.

[35] B. A. Huberman and N. S. Glance, Proc. Natl. Acad. Sci. USA, 90 (1993) 7716.

[36] L. E. Blume, Games Econ. Behav., 5 (1993) 387.

[37] L. E. Blume, Games Econ. Behav., 11 (1995) 111.

[38] G. Szabó and C. Tőke, Phys. Rev. E, 58 (1998) 69.

[39] Z.-X. Wu AND Y.-H. WANG, Phys. Rev. E, 75 (2007) 041114.

[40] X. Chen and L. Wang, Phys. Rev. E, 77 (2008) 017103

[41] Z.-X. Wu And Z. Rong, Phys. Rev. E, 90 (2014) 062102.

[42] A. Traulsen, M. A. Nowak, and J. M. Pacheco, J. Theor. Biol., 244 (2007) 349.

[43] G. Szabó, J. Vukov, and A. Szolnoki, Phys. Rev. E, 72 (2005) 047107.

[44] J. Vukov, G. Szabó, and A. Szolnoki, Phys. Rev. E, 73 (2006) 067103. 